\title{
Location based Routing Protocols: A Survey
}

\author{
Aditya H. Iche \\ Department of Computer Engineering, \\ Sinhgad College of Engineering, \\ Pune, India.
}

\author{
Prof. M R Dhage \\ Department of Computer Engineering, \\ Sinhgad College of Engineering, \\ Pune, India.
}

\begin{abstract}
A Mobile Ad-Hoc Networks (MANET) or Wireless sensor Network (WSN) is a collection of wireless nodes without any centralized access point, topology or centralized administration. Because of this conditions, network overhead and packet delivery ratio get affected. Hence to reduce routing overhead and increase packet delivery ratio in this type network, there are many routing protocols are developed by researchers. Here we are presenting a survey of such routing protocol that make forwarding decision based on node geographic position and route packets only in the direction of destination node. This type of limited flooding reduces the routing overhead in the network. To know the node position, GPS or some other Location services are used.
\end{abstract}

\section{General Terms}

Routing protocol

\section{Keywords}

MANET, WSN, Position Based Routing, Geographic Location

\section{INTRODUCTION}

As we see that in recent year's mobile and wireless technology is growing very rigorously that led to increasing attenuation on mobile ad hoc and sensor network. Different routing strategy are used to route packets in network, some are topology based and some are location based. But by using topology based routing approach in this networks, networks faces many problems, such as more control packet overhead, less packet delivery ratio, end to end delay etc. to solve this problems position based routing approach is proposed.

The rest of this paper are organized as follows. In Section 2 we define the various categories of routing protocols. In sections 3 we discuss the location services and forwarding strategies, section 4 contain different location based routing protocols, section 5 contain comparison of location based routing protocols and lastly we concludes the paper.

\section{VARIOUS CATEGORIES OF ROUTING PROTOCOLS}

According to primary architectural framework, the routing protocols are mainly divided into two types:

\subsection{Topology-based Routing Protocols}

In topology-based routing protocol, for forwarding packets information about the link that are exist in network is used. Furthermore, topology-based routing is dived in Proactive/table driven, reactive/on-demand and hybrid routing approach as shown in figure 1 .

In proactive routing each Mobile node maintains a routing table, which contain destinations and possible path towards destination. These table are periodically broadcast to each node throughout the network. However, the disadvantages is that respective amount of data for maintenance, create lot of traffic, slow reaction on restructuring and failures. An example of proactive routing protocol is OLSR and DSDV [1].

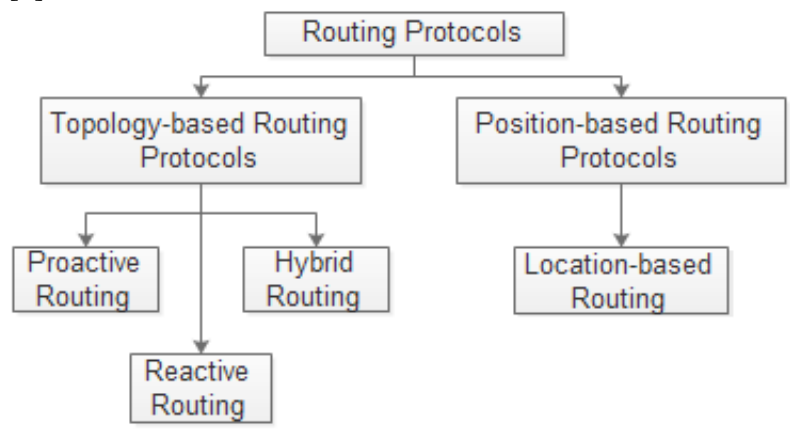

Fig 1: Types of Routing Protocols

Reactive routing protocols maintain only route that are currently in used. The Route are only discover when source mobile node $\mathrm{MN}$ needs to transmit the data packets, therefore reduce the routing overhead in the network. However, reactive routing still have some drawbacks. First, delay in first packet transmission because every time it is typically required to perform rout discovery before exchanging packets. Second, even in route maintenance phase, to keep route available it may generate significant amount of traffic. An example of reactive routing protocol is DSR, TORA and AODV.

Hybrid routing protocol combines advantages of both proactive and reactive protocol.in hybrid routing, usually initiate routing through proactive routing by determining routes and then certain nodes demands are serve according to reactive routing. An example of Hybrid routing protocol is ZRP [1].

\subsection{Position-based Routing Protocol}

Position-based routing protocol uses additional information to overcome the disadvantages in topology-based routing protocol. Here protocol used physical location information of a particular node for communication purpose. Each node aware of its own position, the position of neighbor node within range, and of a destination node through GPS or by some other positioning services. Sender use Location services to determine location of a destination node. Node use broadcast to know location of one hope neighbor. Due to this position-based routing required minimal routing overhead and also avoid delay and latency due to localized forwarding.

\section{LOCATION SERVICES AND FORWARDING STRATEGIES}

In network, location services are used determine the position of a destination node, before sending a packet. The position of neighbor is typically learn by sending beacons to one hop neighbor. These beacons are send periodically by all the nodes. Location services are classified according to how many nodes they services. Node may maintain the position of some specific node or all nodes in the network. Hence there are four 
possible combustions in location services that are, Some for Some, All for Some, Some for All, and lastly All for All [2].

There are three main types of forwarding strategies which are used: Greed forwarding, restricted directional flooding, Hierarchical forwarding.

In greedy packet forwarding, the sender node used the approximate location information of destination node to send the packet using one of the location services. Route establishment and route maintenance phases are not involve in greedy forwarding. When intermediate node receive packets from sender node, it forward the packet to a neighbor lying in the direction of the destination node. The decision is made accordingly to algorithm criteria.

Hierarchical forwarding is used in network which had hierarchical network structure. Here node having maximum energy can be used in processing and sending information while minimum energy node can be used for sensing purpose. Hence by this type of routing energy efficiency is achieved and also increased network lifetime.

In flooding approach every incoming packets are sent to all neighbor in all direction. But in restricted directional flooding, packets are sent to all single hop neighbor towards destination. Neighbor that received packets first check whether they satisfied the criteria to forward the packet or whether they drop they drop the packet.by using this approach we can reduce sufficient routing overhead [2].

\section{LOCATION BASED ROUTING PROTOCOLS}

Young-Bae Ko and Nitin H. Vaidya [2000] have proposed "Location-Aided Routing in mobile ad hoc networks(LAR)" which used location information due to this the search rang of routing of LAR would be restricted, so that the network energy Consumption and routing overhead would reduce. Here, there are two flooding region in LAR protocol, i.e. the expected zone and request zone. When source node wants to send packet to destination node, first source node should get the position of destination node by using location services which is used to get position of node. Two different LAR schemes are presented here: LAR scheme 1 and LAR scheme 2 . In scheme 1 request zone is set to be a rectangular, both the source node and destination node should be included in the region. If the request zone will be the small rectangle, then rang of route searching for node will also be small [3].

"Energy Efficient Location Aided Routing (EELAR) Protocol for Wireless MANETs" have been proposed by Mohammad A. Mikki [2009] which is the modification of basic LocationAided Routing (LAR) protocol. EELAR utilizes location information of nodes with the goal of decreasing routing overhead in MANET. EELAR uses a wireless base station (BS) that covers all mobile nodes (MNs) in the network. BS divides the network into equal six sub-areas. At route discovery phase instead of flooding control packets to the whole network area, in EELAR packets are flooded only in the sub-area where destination mobile node is present. The base station keeps a Position table (PT) that stores locations of all MNs. PT is built by BS through broadcasting small BEACON packets to all MNs in the network. In results author show that EELAR makes significant reduce in energy consumption of network node batteries through limiting the area of discovering a new route to a small zone [4].

Wang et al. [2009] has proposed an efficient greedy location aided routing protocol (GLAR) which improve efficiency of location aided routing protocol in MANATs. Here author decide a baseline, which is line in between source node and the destination node. This baseline is used to broadcast the request packets in request zone for route discovery. The node with shortest distance to baseline is selected as a next hope during broadcast. Here author compare GLAR with basic LAR, and result shows that proposed GLAR can reduce the network overhear and increase network lifetime [5].

P.T.V. Bhuvaneswari et.al. [2010] has proposed A Location Aided Energy Efficient Routing (LAEER) protocol for WSN Here author consider two routing merits for on-demand routing protocol that is location and residual energy of the nodes. Only the forwarding neighbor nodes are involved in routing while other nodes are switched to idle state. Due to considerable reduction in energy consumption in the network can be achieved. To choose optimal forwarding neighbor, protocol used best progress node with suitable link quality which leads to reduction in memory and bandwidth utilization. LAEER protocol contain four main functional modules that are, Localization Engine, Neighborhood Management, Routing Management and lastly Energy Management. When compare to AODV routing protocol author show that LAEER protocol leave behind AODV in terms of control overhead, average end-to-end delay, packet delivery ratio and average energy consumption in the network [6].

Anonymous Location-Aided Routing in Suspicious MANETs (ALARM) proposed by Defrawy et al.[2011] which aimed to provides both security and privacy features, including node authentication, anonymity ,data integrity and intractability in MANATs. ALARM collect node location information and construct a physical MANET map. By using this MANAT map, each node decide which neighbor nodes is to be select to communicate with. ALARM provides both security and privacy features, including node authentication, anonymity, data integrity and intractability [7].

Xiao Hui Li et al. [2011] has proposed a Location-Based SelfAdaptive Routing Algorithm for Wireless Sensor Networks in Home Automation (WSNHA-LBAR). This proposed protocol use cylindrical request zone, hence during route discovery phase, restricted flooding reduces the routing control overhead and decreases broadcast storm problems. Also by using a selfadaptive algorithm based on Bayes theorem, WSNHA-LBAR automatically adjusts the size of the request zone. WSNHALBAR uses the location information of the sensor nodes to restrict the flooding route searching space to a smaller estimated request zone, which represents the route-searched zone. By simulation authors show that WSNHA-LBAR improved network reliability and also reduced routing overhead in network while compare with AODVjr routing protocol [8].

Dan Luo et. al [2011] has an improved hybrid location-based Ad hoc routing protocol (IHLAR) for Ad Hoc Networks. This hybrid approach combines geographic routing with AODV routing protocol. IHLAR overcomes the end-to-end delay problem in reactive routing and also improved the length performance of geographic routing. By Simulation results author shows that routing protocol outperforms the pure reactive routing in terms of average delay and packet delivery rate with compare to other protocols [9].

Jubin Sebastian E et. al. [2012] has proposed a Location Based Opportunistic Routing Protocol for Mobile Ad Hoc Networks (LOR), which helps to the problem of delivering data packets for highly dynamic mobile ad hoc networks in a 
reliable and timely manner. Here to track moving destination nodes, additional check is introduced. At each hop, the node that forwards the packet will check its neighbor list to see whether the destination is within its transmission range. When compare with GPSR and AOMDV routing algorithm author shows that LOR gives better Packet delivery ratio, throughput and lower end to end delay compared with others [10].

F. Gao et. al. [2013] has proposed a Location based Cross layer routing protocol [CLMHR] which is based on basic LAR protocol. Here concept of Cross layering is applied with physical layer which gathered residual battery information and passed it to Network layer for efficient routing. Proposed protocol is increase the network lifetime and also maintain network equilibrium. CLMHR combined three teams, first is equilibrium of candidate relay node's residual energy and then distance from the source node to the destination or next hop and lastly the cross-layer design idea [11].
Haiying Shen et al. [2013] has proposed An Anonymous Location-Based Efficient Routing Protocol [ALERT] for MANETs. The proposed system dynamically dividers the network field into zones and arbitrarily selects nodes in zones as intermediate relay nodes, which form a no traceable anonymous route. It also offers anonymity protection to sources, destinations, and routes by hiding the data initiator among many initiators. When compare to GPSR geographical routing protocol ALERT achieves significant routing efficiency [12]

\section{COMPARISON OF LOCATION BASED ROUTING PROTOCOLS}

The comparison of location based routing protocols is done based on literature survey given in table 1 . The given methods are compared based on type of network, routing overhead, Scalability, end to end delay and security provided in the protocols

Table 1: comparison of location based routing protocols

\begin{tabular}{|c|c|c|c|c|c|c|}
\hline Protocol & N/w type & Overhead & Security & Scalability & $\begin{array}{c}\text { End to end } \\
\text { delay }\end{array}$ & Proactive/Reactive \\
\hline LAR & MANAT & Low & No & Good & Low & Reactive \\
\hline EELAR & MANAT & Low & No & Good & Low & Reactive \\
\hline GLAR & MANAT & Low & No & Good & Low & Reactive \\
\hline LAEER & WSN & Low & No & Good & Low & Reactive \\
\hline ALARM & MANAT & High & Yes & Bad & Low & Reactive \\
\hline WSNHA-LBAR & WSN & Low & No & Good & Low & Reactive \\
\hline IHLAR & Ad Hoc & High & No & Good & Low & Hybrid \\
\hline LOR & MANAT & Low & No & Good & Low & Reactive \\
\hline CLMHR & WSN & Low & No & Good & Low & Reactive \\
\hline ALERT & MANAT & High & YES & Bad & Low & Reactive \\
\hline
\end{tabular}

\section{ACKNOWLEDGMENTS}

I am very thankful to my guide and Head of Computer Engineering Department, Sinhgad College of Engineering Pune, Maharashtra, India for their kind support and constant encouragement for this work.

\section{CONCLUSION}

In this paper we present a survey on location based routing protocols in mobile ad hoc network and in wireless sensor network. From this survey we conclude that, by using node location information and by using restricted flooding approach, we can achieve significant low network overhead as compare to topology based routing approach and get high packet delivery ratio. Also in future by using Cross layer technique that exchange battery information from Physical layer to MAC layer with location based routing may increase network lifetime and also achieve energy efficacy in network.

\section{REFERENCES}

[1] L. K. Qabajeh, L. M. Hiah, M. M. Qabajeh, 2009, “A qualitative comparison of position-based routing protocols for ad-hoc networks", IJCSNS International Journal of Computer Science and Network Security, VOL.9 No.2, February 2009

[2] M Mauve, J Widmer, H Hartenstein, 2001, “A survey on position-based routing in mobile ad hoc networks", Network, IEEE ,Volume:15, Issue: 6.

[3] Young-Bae Ko, Nitin H. Vaidya, 2000, "Location-Aided Routing (LAR) in mobile ad hoc networks", Wireless Networks, vol. 6, no. 4, 2000, pp.307-321.

[4] M. A. Mikki, 2009, "Energy Efficient Location Aided Routing Protocol for Wireless MANETs", (IJCSIS) International Journal of Computer Science and Information Security Vol. 4, No. 1 and 2, 
[5] Neng-Chung Wang, Jong-Shin Chen, Yung-Fa Huang and Si-Ming Wang, "A Greedy Location-Aided Routing Protocol for Mobile Ad Hoc Networks", Proceedings of the 8th WSEAS International Conference on Applied Computer and Applied Computational Science.

[6] P.T.V.Bhuvaneswari and V. Vaidehi, 2010, "Location Aided Energy Efficient Routing Protocol in Wireless Sensor Network", IJSSST, Vol. 11, No. 4.

[7] Karim El Defrawy, Gene Tsudik,, 2011, ALARM: Anonymous Location-Aided Routing in Suspicious MANETs", Transactions on Mobile computing, vol. 10, no. 9, September 2011.

[8] Dan Luo, Jipeng Zhou "An Improved Hybrid LocationBased Routing Protocol for Ad Hoc Networks" 2011 IEEE.

[9] Xiao Hui Li, Seung Ho Hong and Kang Ling Fang, 2011, "Location-Based Self-Adaptive Routing Algorithm for
Wireless Sensor Networks in Home Automation", Systems Volume 2011, Article ID 484690

[10] Jubin Sebastian E , Sreeraj V.R, Tauheed Ul Islam, 2012, "Location Based Opportunistic Routing Protocol for Mobile Ad Hoc Networks", American Jou-01, Issue$01, \mathrm{pp}-16-21$

[11] F. Gao1, Hongli Wen, Lifen Zhao, Yuebin Chen, "Design and Optimization of a Cross-Layer Routing Protocol for Multi-Hop Wireless Sensor Networks", IEEE International Conference on Sensor Network Security Technology and Privacy Communication System, 2013.

[12] Haiying Shen and Lianyu Zhao, 2013, "ALERT: An Anonymous Location-Based Efficient Routing Protocol in MANETs", IEEE transactions on mobile computing, vol. 12, no. 6, June 2013Bowman, M., Debray, S. K., and Peterson, L. L. 1993. Reasoning about naming systems. 